\title{
Stability of an equilibrium state in a multiinfectious-type SIS model on a truncated network
}

\begin{abstract}
In a homogeneous constant population, the basic SIS model potentially has an epidemic equilibrium state with global asymptotic stability since it can be reduced to the logistic equation. On the basic SIS model with a nonhomogeneous constant population, viewed as a multitype SIS model, the global or local asymptotic stability of an epidemic equilibrium state has also been studied. ${ }^{1-4}$ However, this kind of analysis in other models with nonhomogeneous populations has rarely been developed, even though the corresponding models with homogeneous populations are well known. In addition, recent studies of complex networks have revealed that heterogeneity of the link number of vertices drastically changes the epidemic thresholds. ${ }^{5-9}$ For these reasons, figuring out the roles of heterogeneity is a major topic in epidemic modeling. Here, we consider a multiinfectious-type SIS model on a network, and show the (local or global) asymptotic stability of an epidemic equilibrium state whenever it exists.
\end{abstract}

Key words Multiinfectious-type SIS model $\cdot$ Permanence Asymptotic stability

\section{Introduction}

Epidemic processes are complicated in terms of interactions between both individuals and social networks. In addition, heterogeneity at various levels, for example, the characteristics of pathogens, infective agents, and regions of outbreak, would prevent refined data analysis or mathematical

N. Sugimine $(\square) \cdot K$. Aihara

Aihara Complexity Modelling Project, ERATO, JST, 3-23-5-201

Uehara, Shibuya-ku, Tokyo 151-0064, Japan

Tel. +81-3-5452-0371; Fax +81-3-5452-0377

e-mail: sugimine@aihara.jst.go.jp

N. Sugimine $\cdot$ K. Aihara

Institute of Industrial Science, University of Tokyo, Tokyo, Japan

This work was presented in part at the 11th International Symposium of Artificial Life and Robotics, Oita, Japan, January 23-25, 2006 modeling to clarify epidemiologically infectious diseases in order to control the spread of infection. To give a little help to this problem, we consider a simple deterministic epidemic model given by ordinary differential equations. For this, expressions of the effects of networks in differential equations are essential, since several well-studied simple epidemic models, such as the SIS, SIR, and SEIR models, have been used to study infectious diseases for a single homogeneous group., ${ }^{3,10-12}$ Here, S, I, R, and E mean the susceptible, the infectious, the removal, and the exposed, respectively. Furthermore, the difference in the threshold forms for epidemics caused by the effects of networks is also important.

In this direction, Pastor-Satorras and Vespignani ${ }^{5}$ first succeeded in studying SIS models on scale-free networks by large-scale simulations and analytical methods based on the mean-field approximations. The scale-free property is one common aspect that real networks possess quite often, as determined by studies of complex networks over the last decade. Scale-free networks can be generated by random procedures with the preference attachment. ${ }^{6,13}$ Power-law distributions of vertex degrees make possible a nonnegligible density of vertices with very high degrees in such networks. These factors could encourage the mean-field approximation on scale-free networks. This would be one reasons for Pastor-Satorras and Vespignani's success. They considered a version of SIS models that reflect the scale-free property, and showed that the epidemic threshold of the infection rate is zero if vertex degree distributions with finite means do not have quadratic moments. In other words, an epidemic equilibrium solution exists on such networks unless the infection rate is zero. Thus, an epidemic equilibrium state always exists on scale-free networks with indices between 2 and 3, which are typically observed in all real networks investigated with power-law vertex degree distributions. After this first success, more details of these models were investigated. ${ }^{6}$ Several authors ${ }^{7-9}$ showed that epidemics always occur in various SIS-type models on the same networks as described by Pastor-Satorras and Vespignani. ${ }^{5}$

From a mathematical aspect, such SIS-type models on networks are viewed as multi-type SIS models ${ }^{1,2}$ (see 
also Sect. 8 in Rass and Radcliffe ${ }^{3}$ ) if the networks possess the bounded degree property. Further, exact calculations to obtain the epidemic thresholds in such models can be carried out in an analysis for the multi-type SIS model. The restriction of the bounded degree property is not so serious in such applications, since real networks are finite ones even if they can be approximated by scale-free networks.

As considered elsewhere, ${ }^{9,14,15}$ we are also interested in situations where the majority cannot sustain epidemics without the presence of the core of individuals who potentially become infectious with large infection rates. For example, for the prevalence of sexually transmitted diseases, the core would indicate individuals engaging in risky sexual behavior, ${ }^{14}$ and in SARS, the core would mean individuals called superspreaders. ${ }^{15}$ Hence, we consider a population with two types of individual: the majority who potentially have a small infection rate, and the core who potentially have a large one. To make the calculation for stability analysis tractable, we only consider the susceptible individuals and the two types of infectious individuals with no immunities, and we do not take into account the latency period and the incubation period. We assume that infected individuals soon become infectious and have a small infection rate with probability $(1-p)$ or a large one with probability $p$. In this article, we introduce such a two-infectious-type SIS model on truncated networks, and show that either the permanence of an epidemic equilibrium state or the global asymptotic stability of a disease-free equilibrium state holds. We also show that if the majority and the core have the same recovery rate, either an epidemic equilibrium state or a disease-free one is globally asymptotically stable.

\section{Model and results}

We introduce the following assumption on vertex degree distributions in networks, which is widely accepted in the field of complex networks ${ }^{6}$ (see also Sect. 10.5 in Diekmann and Heesterbeek ${ }^{5}$ ).

Assumption 1: Let $k$ be a positive integer. We define $p(k)$ to be the probability that a vertex chosen uniformly at random has vertex degree $k$. Then, the probability $p(j \mid k)$ that a given vertex with degree $k$ is linked to a vertex with degree $j$ by one edge is proportional to $j p(j)$, which is independent of its own vertex degree $k$. In other words, whenever $p(k)>0$,

$p(j \mid k)=\frac{j p(j)}{\sum_{j^{\prime}} j^{\prime} p\left(j^{\prime}\right)}$

holds independently of $k$.

Further, we need the following technical assumption for the mathematical analysis.

Assumption 2: There exists some positive integer $K$ such that $p(k)=0$ for every $k \geq K+1$.
For simplicity, we only consider the case where $p(k)>0$ for all $1 \leq k \leq K$. By noting that Eq. 2 should be omitted for $k$ with $p(k)=0$, we can adapt our proofs to the case where $p(k)=0$ for some $1 \leq k \leq K$. For simplicity of notation, we denote by $\langle f\rangle$ the expectation of $f$ with respect to $\{p(j)\}_{k=1}^{K}$, and define $q(k)=k p(k) /\langle j\rangle$ for every $1 \leq k \leq K$.

We consider a population with two types of individual where the majority has the small infection rate $\lambda_{1}$ and the core has the large one $\lambda_{2}$. For this purpose, we treat three relative densities $s_{k}, u_{k}$, and $v_{k}$ of vertices with degree $k ; s_{k}$, $u_{k}$, and $v_{k}$ are the densities of the susceptible, the infectious majority, and the infectious core, respectively. If we treat more than two types, we can also obtain similar results. This is why we call the following Eq. 2 a multiinfectious-type SIS model. Following Pastor-Satorras and Vespignani, ${ }^{5}$ we consider the dynamics of $\left\{\left(s_{k}, u_{k}, v_{k}\right)\right\}_{k=1}^{K}$ given by

$$
\begin{aligned}
& \frac{\mathrm{d} s_{k}}{\mathrm{~d} t}(t)=u_{k}(t)+\delta v_{k}(t)-k s_{k}(t)(U(t)+V(t)) \\
& \frac{\mathrm{d} u_{k}}{\mathrm{~d} t}(t)=-u_{k}(t)+k s_{k}(t)(U(t)+V(t))(1-p) \\
& \frac{\mathrm{d} v_{k}}{\mathrm{~d} t}(t)=-\delta v_{k}(t)+k s_{k}(t)(U(t)+V(t)) p
\end{aligned}
$$

where $\delta>0, p \in(0,1)$,

$U(t)=\lambda_{1} \sum_{j} q(j) u_{j}(t)$, and $\quad V(t)=\lambda_{2} \sum_{j} q(j) v_{j}(t)$

Although $U(t)$ and $V(t)$ should be represented by

$U_{k}(t)=\lambda_{1} \sum_{j} p(j \mid k) u_{j}(t) \quad$ and $\quad V_{k}(t)=\lambda_{2} \sum_{j} p(j \mid k) v_{j}(t)$

respectively, a simpler version of Eq. 2 can be used because of Eq. 1.

Let us define $\Omega$ as follows:

$\Omega=\left\{\left\{\left(x_{k}, y_{k}, z_{k}\right)\right\}_{k=1}^{K}: \begin{array}{c}\text { for every } 1 \leq k \leq K, \\ x_{k}, y_{k}, z_{k} \geq 0 \text { and } \\ x_{k}+y_{k}+z_{k}=1\end{array}\right\}$

The dynamics $\left\{\left\{\left(s_{k}(t), u_{k}(t), v_{k}(t)\right)\right\}_{k=1}^{K} ; t \geq 0\right\}$ satisfy the permanence if

$\liminf _{t \rightarrow \infty}\left\|\left\{\left(u_{k}(t), v_{k}(t)\right)\right\}_{k=1}^{K}\right\| \geq \varepsilon$

for some $\varepsilon>0$ and any initial state in $\Omega \backslash\{(1,0,0)\}_{k-1}^{K}$, where $\|\cdot\|$ stands for the $2 K$-dimensional Euclidean norm.

Theorem 1: (i) There exists a unique epidemic equilibrium solution of Eq. 2 belonging to $\Omega \backslash\{(1,0,0)\}_{k=1}^{K}$ if and only if

$\left(\lambda_{1}(1-p)+\lambda_{2} \frac{p}{\delta}\right) \frac{\left\langle k^{2}\right\rangle}{\langle k\rangle}>1$

Such a solution $\left\{\left(s_{k}, u_{k}, v_{k}\right)\right\}_{k+1}^{K}$ satisfies $u_{k}, v_{k}>0$ for all $1 \leq k \leq K$. 
(ii) If Eq. 3 holds, Eq. 2 satisfies the permanence. Otherwise, a disease-free equilibrium solution $\{(1,0,0)\}_{k=1}^{K}$ of Eq. 2 is globally asymptotically stable.

As shown in the proof below, part (i) of this theorem can be obtained by a slightly modified argument in Pastor Satorras and Vespignani ${ }^{5}$ (see also Masuda and Konno ${ }^{9}$ ), and part (ii) of this theorem is an application of Theorem 4.2 in Lajmanovich and Yorke. ${ }^{1}$

Proof: Any equilibrium solution $\left\{\left(s_{k}, u_{k}, v_{k}\right)\right\}_{k=1}^{K}$ of Eq. 2 satisfies that for all $1 \leq k \leq K$,

$$
\begin{aligned}
& s_{k}=\frac{1}{1+k W\left(1-(\delta-1) p^{*}\right)}, \quad u_{k}=k s_{k} W(1-p), \\
& \text { and } v_{k}=k s_{k} W p^{*}
\end{aligned}
$$

where $W=\Sigma_{j} q(j)\left(\lambda_{1} u_{j}+\lambda_{2} v_{j}\right)$, and $p^{*}=p / \delta$. Let $\lambda^{*}=$ $\lambda_{1}(1-p)+\lambda_{2} p^{*}$. From Eq. 4 ,

$W=\frac{\lambda^{*}}{\langle k\rangle}\left\langle k^{2} \frac{W}{1+k W\left(1-(\delta-1) p^{*}\right)}\right\rangle$

Since the right-hand side of Eq. 5 consists of the increasing concave functions of $W$, Eq. 5 has a unique positive solution $W$ if and only if Eq. 3 holds. From this and Eq. 4, the dynamics of Eq. 2 has a unique equilibrium solution $\left\{\left(s_{k}, u_{k}, v_{k}\right)\right\}_{k+1}^{K}$ in $\Omega \backslash\{(1,0,0)\}_{k=1}^{K}$ such that $u_{k}, v_{k}>0$ for all $1 \leq k \leq K$ if and only if Eq. 3 holds.

Next, we show part (ii) of Theorem 1. Note that Eq. 2 can be rewritten as

$$
\begin{aligned}
\frac{\mathrm{d} u_{k}}{\mathrm{~d} t}(t) & =-u_{k}(t)+k\left(1-u_{k}(t)-v_{k}(t)\right)(U(t)+V(t))(1-p) \\
\frac{\mathrm{d} v_{k}}{\mathrm{~d} t}(t) & =-\delta v_{k}(t)+k\left(1-u_{k}(t)-v_{k}(t)\right)(U(t)+V(t)) p
\end{aligned}
$$

Remembering that $U(t)=\lambda_{1} \Sigma_{j} q(j) u_{j}(t)$ and $V(t)=$ $\lambda_{2} \Sigma_{j} q(j) v_{j}(t)$. By Theorem 4.2 in Lajmanovich and Yorke, ${ }^{1}$ we can obtain part (ii) of Theorem 1 once we examine the real parts of all eigenvalues of

$\left.\begin{array}{cccc}f_{1}(1)-1 & f_{2}(1) & \ldots & \multicolumn{2}{l}{f_{1}(j)} \\ g_{1}(1) & g_{2}(1)-\delta & \ldots & g_{1}(j) \\ \vdots & \vdots & \vdots & \vdots \\ j f_{1}(1) & j f_{2}(1) & \ldots & j f_{1}(j)-1 \\ j g_{1}(1) & j g_{2}(1) & \ldots & j g_{1}(j) \\ \vdots & \vdots & \vdots & \vdots \\ K f_{1}(1) & K f_{2}(1) & \ldots & K f_{1}(j) \\ K g_{1}(1) & K g_{2}(1) & \ldots & k g_{1}(j) \\ f_{2}(j) & \ldots & f_{1}(K) & f_{2}(K) \\ g_{2}(j) & \ldots & g_{1}(K) & g_{2}(K) \\ \vdots & \vdots & \vdots & \vdots \\ j f_{2}(j) & \ldots & j f_{1}(K) & j f_{2}(K) \\ j g_{2}(j)-\delta & \ldots & j g_{1}(K) & j g_{2}(K) \\ \vdots & \vdots & \vdots & \vdots \\ K f_{2}(j) & \ldots & K f_{1}(K)-1 & K f_{2}(K) \\ K g_{2}(j) & \ldots & K g_{1}(K) & K g_{2}(K)-\delta\end{array}\right)$

where $f_{i}(j)=\lambda_{i} q(j)(1-p)$ and $g_{i}(j)=\lambda_{i} q(j) p$ for each $1 \leq j$ $\leq K$ and $i=1,2$. We denote the above matrix by $\mathbf{A}$. Assume that $\mathbf{A} \omega=\kappa \omega$ for some real number $\kappa$ and vector $\omega$. Then such a real number $\kappa$ is characterized by

$\frac{\left\langle k^{2}\right\rangle}{\langle k\rangle}\left(\frac{\lambda_{1}(1-p)}{\kappa+1}+\frac{\lambda_{2} p}{\kappa+\delta}\right)=1$

Therefore, there exists a unique positive eigenvalue of $\mathbf{A}$ if Eq. 3 holds; otherwise all real-valued eigenvalues of $\mathbf{A}$ are nonpositive. By the Perron-Frobenius theorem, this implies that the maximal of the real parts of all eigenvalues of $\mathbf{A}$ is positive if and only if Eq. 3 holds.

Theorem 2: Suppose that Eq. 3 holds. Then for $\delta$ sufficiently close to 1 , the solution $\left\{\left(s_{k}, u_{k}, v_{k}\right)\right\}_{k=1}^{K}$ in Theorem 1 is locally asymptotically stable. Moreover, the solution $\left\{\left(s_{k}, u_{k}, v_{k}\right)\right\}_{k=1}^{K}$ is globally asymptotically stable in $\Omega \backslash\{(1,0,0)\}_{k+1}^{K}$ when $\delta=1$.

Proof: We first prove the local asymptotic stability of $\left\{\left(s_{k}, u_{k}, v_{k}\right)\right\}_{k=1}^{K}$. We consider the linearized dynamics of Eq. 2 at $\left\{\left(s_{k}, u_{k}, v_{k}\right)\right\}_{k=1}^{K}$.

$$
\begin{aligned}
& \frac{\mathrm{d} \tilde{s}_{k}}{\mathrm{~d} t}(t)=\tilde{u}_{k}(t)+\delta \tilde{v}_{k}(t)-k\left(s_{k}(t) \tilde{W}(t)+W \tilde{s}_{k}(t)\right) \\
& \frac{\mathrm{d} \tilde{u}_{k}}{\mathrm{~d} t}(t)=-\tilde{u}_{k}(t)+k(1-p)\left(s_{k} \tilde{W}(t)+W \tilde{s}_{k}(t)\right) \\
& \frac{\mathrm{d} \tilde{v}_{k}}{\mathrm{~d} t}(t)=-\delta \tilde{v}_{k}(t)+k p\left(s_{k} \tilde{W}(t)+W \tilde{s}_{k}(t)\right)
\end{aligned}
$$

where $\tilde{W}(t)=\Sigma_{j} q(j)\left(\lambda_{1} \tilde{u}_{j}(t)+\lambda_{2} \tilde{v}_{j}(t)\right)$. From now on, we set $\delta=1$ and $K$ to be odd. We can similarly treat the case where $K$ is even. Let $a_{j}=\lambda^{*} W j q(j)$. Since we obtain from Eqs. 4 and 6 that

$$
\frac{\mathrm{d} \tilde{W}}{\mathrm{~d} t}(t)=\frac{\lambda^{*} W}{\langle k\rangle}\left(\sum_{k} k^{2} p(k) \tilde{s}_{k}(t)\right)
$$

we consider the linear dynamics

$$
\frac{\mathrm{d}}{\mathrm{d} t}\left(\begin{array}{c}
\tilde{W}(t) \\
\tilde{s}_{1}(t) \\
\vdots \\
\tilde{s}_{K}(t)
\end{array}\right)=\mathrm{M}\left(\begin{array}{c}
\tilde{W}(t) \\
\tilde{s}_{1}(t) \\
\vdots \\
\tilde{s}_{K}(t)
\end{array}\right)
$$

where

$$
\mathbf{M}=\left(\begin{array}{cccccc}
0 & a_{1} & \ldots & a_{j} & \ldots & a_{K} \\
-s_{1} & -1-W & 0 & \ldots & \ldots & 0 \\
\vdots & 0 & \ddots & \ddots & & \vdots \\
-j s_{j} & \vdots & \ddots & 1-j W & \ddots & \vdots \\
\vdots & \vdots & & \ddots & \ddots & 0 \\
-K s_{k} & 0 & \ldots & \ldots & 0 & -1-K W
\end{array}\right)
$$

We will show that the real parts of all eigenvalues of $\mathbf{M}$ are negative. Let $c_{j}=j s_{j} a_{j}$. The characteristic equation $\Phi_{\mathbf{M}}$ of $\mathbf{M}$ is

$$
\begin{aligned}
\Phi_{\mathbf{M}}(x)= & x(x+1+W) \ldots(x+1+K W) \\
& +c_{1}(x+1+2 W) \ldots(x+1+K W) \\
& +c_{2}(x+1+W)(x+1+3 W) \ldots(x+1+K W) \\
& \vdots \\
& +c_{K}(x+1+W) \ldots(x+1+(K-1) W)
\end{aligned}
$$


Equation 8 of $\Phi_{\mathbf{M}}(x)$ shows that

$\Phi_{\mathbf{M}}(0)>0, \quad \lim _{x \rightarrow \infty} \Phi_{\mathbf{M}}(x)=\infty$

and the coefficient of degree $K$ is

$\sum_{j=1}^{K}(1+j W)$

Equation 8 of $\Phi_{\mathbf{M}}(x)$ also shows that $\Phi_{\mathbf{M}}(-1-j W) \Phi_{\mathbf{M}}(-1-$ $(j-1) W)<0$ for every $2 \leq j \leq K$, which implies that for every $2 \leq j \leq K$, there exists at least one root $-\alpha_{j}$ of the equation $\Phi_{\mathbf{M}}(x)+0$ in $(-1-j W,-1-(j-1) W)$. Then, $\Phi_{\mathbf{M}}(x)$ $=\left(x+\alpha_{2}\right) \ldots\left(x+\alpha_{K}\right)\left(x^{2}+\beta x+\gamma\right)$ for some real numbers $\beta$ and $\gamma$. In this expression, the coefficient of degree $K$ is

$\sum_{j=2}^{K} \alpha_{j}+\beta$

From Eqs. 10 and 11 together with $\alpha_{j}<1+j W$, it follows that $\beta>0$. When the equation

$x^{2}+\beta x+\gamma=0$

has no real-valued solutions, the real parts $-\beta / 2$ of the two solutions are negative. Suppose that equation Eq. 12 has two real-valued solutions. Then, by $\beta>0$ and Eq. 9, both the solutions are negative. Therefore, we have shown that the real parts of all eigenvalues of $\mathbf{M}$ are negative. In other words, the linear dynamics Eq. 7 is asymptotically stable.

Recall that we considered the case where $\delta=1$ in the linearized dynamics of Eq. 6 . By the asymptotic stability of $\left(\tilde{W}(t), \tilde{s}_{1}(t), \ldots, \tilde{s}_{K}(t)\right)$, the second and third equations of Eq. 6 imply that the linearized dynamics of Eq. 6 is also asymptotically stable, which is equivalent to the real parts of all eigenvalues of the corresponding matrix to Eq. 6 being negative. Thus, by continuity, the dynamics of Eq. 2 is locally asymptotically stable at $\left\{\left(s_{k}, u_{k}, v_{k}\right)\right\}_{k=1}^{K}$ for $\delta$ sufficiently close to 1 .

To obtain the global asymptotic stability of $\left\{\left(s_{k}, u_{k}, v_{k}\right)\right\}_{k=1}^{K}$ for $\delta=1$, we need a slight modification to the earlier argument. ${ }^{1-3}$ The key ingredients are as follows:

For every $1 \leq k \leq K$,

$p u_{k}(t)=(1-p) v_{k}(t)+w_{k} e^{-t}$

and

$p s_{k}(t)+v_{k}(t)=p-w_{k} e^{-t}$

where $w_{k}=p u_{k}(0)-(1-p) v_{k}(0)$.

For a while, we assume that $w_{k}=0$ for all $1 \leq k \leq K$. Then from Eqs. 2, 13, and 14, we have that

$$
\begin{aligned}
\frac{\mathrm{d} v_{k}}{\mathrm{~d} t}(t) & =-v_{k}(t)+\lambda * k s_{k}(t) \sum_{j} q(j) v_{j}(t) \\
& =-v_{k}(t)+\frac{k \lambda^{*}}{p}\left(p-v_{k}(t)\right) \sum_{j} q(j) v_{j}(t)
\end{aligned}
$$

This shows that the system $\left\{\left(p s_{k}(t), v_{k}(t)\right)\right\}_{k=1}^{K}$ in $[0, p]^{2 K}$ is reduced to the SIS model considered in Lajmanovich and Yorke. ${ }^{1}$ Thus, the equilibrium solution $\left\{\left(p s_{k}, v_{k}\right)\right\}_{k=1}^{K}$ of Eq. 15 is global asymptotically stable in $[0, p]^{2 K}$ $\{(p, 0)\}_{k=1}^{K}$.

In general cases, from Eqs. 2, 13, and 14,

$$
\begin{aligned}
\frac{\mathrm{d}}{\mathrm{d} t} v_{k}(t)= & -v_{k}(t)+k s_{k}(t)\left(\lambda * \sum_{j} q(j) v_{j}(t)+\lambda_{1}\left\langle w_{j}\right\rangle_{q} e^{-t}\right) \\
= & -v_{k}(t)+\frac{k \lambda^{*}}{p}\left(p-w_{k} e^{-t}-v_{k}(t)\right) \sum_{j} q(j) v_{j}(t) \\
& +k \lambda_{1}\left\langle w_{j}\right\rangle_{q} e^{-t} s_{k}(t)
\end{aligned}
$$

where $\langle\cdot\rangle_{q}$ denotes the expectation with respect to $\{q(j)\}_{j=1}^{K}$. For a function $\Phi$ on $\mathbb{R}^{K}$ and a semiflow $\{\phi(t)\}_{t \geq 0}$ in $\mathbb{R}^{K}$, let

$\Phi_{+, u}^{\prime}(\phi(t))=\limsup _{h \searrow 0} \frac{\Phi(\phi(t+h))-\Phi(\phi(t))}{h}$

Let $v(t)=\left(v_{1}(t), \ldots, v_{K}(t)\right)$. Define

$M(v(t))=\max _{1 \leq j \leq K} \frac{v_{j}(t)}{v_{j}}$

For $\tau>0$, we can assume without loss of generality that there exist some $1 \leq k \leq K$ and $\eta>0$ such that for all $t \in[\tau, \tau+\eta]$

$M(v(t))=\frac{v_{k}(t)}{v_{k}}$

Note that $v_{i}(\tau) \leq\left(v_{i} / v_{k}\right) v_{k}(\tau)$ for every $j \neq k$. Suppose that $M(v(\tau)) \geq 1+\varepsilon$ for a given $\varepsilon>0$. Then,

$$
\begin{aligned}
\frac{\mathrm{d}}{\mathrm{d} t} v_{k}(\tau) \leq & \frac{v_{k}(\tau)}{v_{k}}\left(-v_{k}+\frac{k \lambda^{*}}{p}\left(p-w_{k} e^{-\tau}-v_{k}(1+\varepsilon)\right)\left\langle v_{j}\right\rangle_{q}\right) \\
& +k \lambda_{1}\left\langle w_{j}\right\rangle_{q} e^{-\tau} s_{k}(\tau) \\
= & \frac{v_{k}(\tau)}{v_{k}}\left(-v_{k}+\frac{k \lambda^{*}}{p}\left(p-v_{k}\right)\left\langle v_{j}\right\rangle_{q}\right. \\
& \left.-\frac{k \lambda^{*}}{p}\left(\varepsilon v_{k}+w_{k} e^{-\tau}\right)\left\langle v_{j}\right\rangle_{q}\right)+k \lambda_{1}\left\langle w_{j}\right\rangle_{q} e^{-\tau} s_{k}(\tau) \\
\leq & -\frac{k \lambda^{*}}{p}\left(\varepsilon v_{k}+w_{k} e^{-\tau}\right)\left\langle v_{j}\right\rangle_{q}+k \lambda_{1}\left\langle w_{j}\right\rangle_{q} e^{-\tau} s_{k}(\tau)
\end{aligned}
$$

Here, in the third inequality we use

$$
\begin{aligned}
-v_{k} & +\frac{k \lambda^{*}}{p}\left(p-v_{k}\right)\left\langle v_{j}\right\rangle_{q} \\
& =-v_{k}+k s_{k}\left(\lambda_{1} \sum_{j} q(j) u_{j}+\lambda_{2} \sum_{j} q(j) v_{j}\right) p=0
\end{aligned}
$$

which follows from the fact that $p u_{k}=(1-p) v_{k}$ and $p s_{k}+v_{k}$ $=p$ hold for every $1 \leq k \leq K$. From Eq. 17, we obtain, independently of $\left\{w_{k}\right\}_{k=1}^{K}$, that for large enough $\tau$,

$$
M_{+, u}^{\prime}(v(t))<0
$$

if $M(v(\tau)) \geq 1+\varepsilon$. Define $m(v(t))=\min _{1 \leq j \leq K}\left\{v_{j}(t) / v_{j}\right\}$. If $m(v(t))$ $\leq 1-\varepsilon$, then by a similar argument as in Eqs. 16-18, we obtain, independently of $\left\{w_{k}\right\}_{k=1}^{K}$, that for large enough $\tau$, 
$m_{+, u}^{\prime}(v(t))>0$

From Eqs. 18 and 19, we have that for sufficiently large $t$ and all initial conditions in $\Omega \backslash\{(1,0,0)\}_{k=1}^{K}$,

$V_{+, u}^{\prime}(v(t)) \leq 0, \quad$ and $\quad W_{+, u}^{\prime}(v(t)) \leq 0$

where $V(z)=\max \{M(z)-(1+\varepsilon), 0\}$ and $W(z)=\max \{(1-\varepsilon)$ $-m(z), 0\}$. Let $H_{V}=\left\{z: V_{+, u}^{\prime}(z)=0\right\}$ and $H_{W}=\left\{z: W_{+, u}^{\prime}(z)=\right.$ $0\}$. Then we can see from Eqs. 18-20 that $H_{V} \subset\left\{z: 0 \leq z_{j} \leq\right.$ $(1+\varepsilon) v_{j}$ for all $\left.1 \leq j \leq K\right\}$ and $H_{W} \subset\left\{z:(1-\varepsilon) v_{j} \leq z_{j} \leq 1\right.$ for all $1 \leq j \leq K\} \cup\{0\}$, which implies that

$$
H_{V} \cap H_{W} \subset\left\{z:(1-\varepsilon) v_{j} \leq z_{j} \leq(1+\varepsilon) v_{j} \text { for all } 1 \leq j \leq K\right\} \cup\{0\}
$$

Since $\varepsilon>0$ can be arbitrary small, this, together with Eqs. 13, 14, and 20 and Theorem 1, shows the global asymptotic stability of $\left\{\left(s_{k}, u_{k}, v_{k}\right)\right\}_{k=1}^{K}$ in $\Omega \backslash\{(1,0,0)\}_{k=1}^{K}$.

\section{Discussion}

We have introduced a new way to understand the epidemic threshold formula of Eq. 3 on truncated networks ${ }^{5,7-9}$ on the basis of Lajmanovich and Yorke's early work ${ }^{1}$ and its extension $^{2}$ (see also Sect. 8 in Rass and Radcliffe ${ }^{3}$ ). This method can also be applied to most models studied by Masuda and Konno. ${ }^{9}$ Although we have only considered the case where vertex degree distributions are truncated, we think that this does not matter in applications for real networks because of the finiteness of the world. Furthermore, from the viewpoint of rigorous mathematical analysis, we would need to handle the divergence problem to obtain the epidemic threshold formula in Eq. 3 if we treated networks with an infinite quadratic moment like scale-free networks whose indices are less than or equal to 3. Since the late 1990s, many real networks have been recognized to have the scale-free property that can lead the divergence of the quadratic moment $\left\langle j^{2}\right\rangle$ of their vertex degree distributions. When $\left\langle j^{2}\right\rangle$ is large, according to Eq. 3 epidemic thresholds become small. Thus, even if epidemics do not occur, they might be suppressed by a narrow margin. For this reason together with part (ii) of Theorem 1, a small increase in the proportion of core members could cause epidemics even if all new individuals corresponding to that small increase are susceptible. In particular, when $\delta=1$, Theorem 2 indicates that all individuals face the threat of infection even if some of them have a small degree of connectivity. This would suggest that decreasing the proportion of core members at any time can be a useful strategy.

Acknowledgment We would like to thank Prof. H. Inaba for his helpful comments on our work.

\section{References}

1. Lajmanovich A, Yorke JA (1976) A deterministic model for gonorrhea in a nonhomogeneous population. Math Biosci 28: 221-236

2. Rass L, Radcliffe J (2000) Global asymptotic convergence results for multitype models. Int J Appl Math Comput Sci 10:63-79

3. Rass L, Radcliffe J (2003) Spatial deterministic epidemics. American Mathematical Society, Providence

4. Olinky R, Stone L (2004) Unexpected epidemic thresholds in heterogeneous networks: the role of disease transmission. Phys Rev E 70:030902

5. Pastor-Satorras R, Vespignani A (2001) Epidemic spreading in scale-free networks. Phys Rev Lett 86:3200-3203

6. Newman MEJ (2003) The structure and function of complex networks. SIAM Rev 45:167-256

7. Joo J, Lebowitz JL (2004) Behavior of susceptible-infectedsusceptible epidemics on heterogeneous networks with saturation. Phys Rev E 69:066105

8. Liu J, Wu J, Yang ZR (2004) The spread of infectious disease on complex networks with household structure. Physica A 341:273280

9. Masuda N, Konno N (2006) Multi-state epidemic processes on complex networks. J Theor Biol 243:64-75

10. Anderson RM, May RM (1991) Infectious diseases of humans. Oxford University Press, Oxford

11. Diekmann O, Heesterbeek JAP (2000) Mathematical epidemiology of infectious diseases: model building, analysis and interpretation. Wiley, Chichester

12. Thieme HR (2003) Mathematics in population biology. Princeton University Press, Princeton, Oxford

13. Smythe RT, Mahmoud HM (1995) A survey of recursive trees. Theor Prob Math Statist 51:1-27

14. Schinazi RB (2001) On the importance of risky behavior in the transmission of sexually transmitted diseases. Math Biosci 173: 25-33

15. Masuda N, Konno N, Aihara K (2004) Transmission of severe acute respiratory syndrome in dynamical small-world networks. Phys Rev E 69:031917 